\title{
Possible role of the antibiotic-modified microbiome in the development of hematological malignancies in European countries
}

\author{
Gábor Ternák*1, Károly Berényi², Balázs Németh² ${ }^{2}$ Ágnes Szenczi ${ }^{2}$, István Kiss²
}

${ }^{*}$ Corresponding author: Gábor Ternák, ${ }^{1}$ University of Pécs, Medical School, Institute of Migration Health, Pécs, Szigeti st. 12., H-7624 Hungary, e-mail: gabor.ternak@aok.pte.hu

Károly Berényi ${ }^{2}$ University of Pécs, Department of Public Health Medicine, Szigeti st. 12., H-7624 Hungary karoly.berenyi@aok.pte.hu

Balázs Németh, ${ }^{2}$ University of Pécs, Department of Public Health Medicine, Szigeti st. 12., H-7624 Hungary balazs.nemeth@aok.pte.hu

Ágnes Szenczy ${ }^{2}$ University of Pécs, Department of Public Health Medicine, Szigeti st. 12., H-7624 Hungary agnes.szenczi@aok.pte.hu István Kiss ${ }^{2}$ University of Pécs, Department of Public Health Medicine, Szigeti st. 12., H-7624 Hungary istvan.kiss@aok.pte.hu

Key words: Hematological malignancies, Hodgkin lymphoma (HL), Non-Hodgkin Lymphoma (NHL), Multiple myeloma (MM), Leukemia (LEU), antibiotics, microbiome, dysbiosis

\begin{abstract}
Hematological malignancies are considered the fifth most common cancer in the world. Several risk factors and probable etiological agents have been suspected in the pathomechanism of those malignancies as infections, chemicals, irradiation, etc., and recently, the contribution of the altered gut flora, dysbiosis, was identified also as a possible additional factor to the existing ones. Host, and external factors, like antibiotics, which were identified as a major disruptor of the "normal" gut flora, influence the composition of the microbiome. Considering the several-fold differences in antibiotic consumption patterns and the incidence of hematological malignancies in European countries, the hypothesis was raised that the dominant consumption of certain antibiotic classes might influence the incidence of different hematological malignancies through the modification of gut flora. Comparisons were performed between the average antibiotic consumption databases reported yearly by ECDC (2009-2019) and the incidence rate of Hodkin lymphoma (HL), non-Hodgkin lymphoma (NHL), multiple myeloma (MM), and leukemia (LEU) estimated for 2020 in 30 European countries. Applying Spearman calculations, significant positive correlation has been found between the incidence of $\mathrm{HL}$ and tetracycline (Jo1A) consumption ( $r=0.399, p=0,029), \mathrm{NHL}$ and narrow spectrum, beta-lactamase resistant penicillin ( $\mathrm{JO1CF})(r=0.580, p=0,001), \mathrm{MM}$ and tetracycline $(r=$ $0.492, p=0.006)$, penicillin ( $\mathrm{J01C})(r=0.366, p=0.047)$, narrow spectrum, beta-lactamase resistant penicillin ( $\mathrm{Jo1} \mathrm{CF})(r=0.574, p=0.001)$, while strong, significant negative correlation has been recorded between NHL and cephalosporin $(r=-0,460, p=0,011)$, and quinolone $(r=-0,380, p=0,038)$. The incidence of LEU did not show any positive or negative association with any antibiotic classes. It is concluded that certain antibiotic classes, in addition to other putative factors, might promote or inhibit the development of different hematological malignancies.
\end{abstract}

\footnotetext{
Abbreviations

ECDC European Centre for disease prevention and control

SDI Sociodemographic Index

HMs Hematological malignancies

HL Hodgkin Lymphoma

NHL Non-Hodgkin Lymphoma
} 
MM Multiple Myeloma

LEU Leukemia

ECIS European Cancer Information System

Cancer cases increased by 28\% between 2006 and 2016, but disparity has been observed between different countries with low and high Sociodemographic Index (SDI), and the smallest increase was seen in high SDI countries, but despite the rapidly increasing cancer burden in lower SDI countries, the probability of developing cancer (age-standardized rates) are still higher in countries of higher SDI. Notable exceptions are cancers with infectious etiologies like cervical (Human Papilloma Virus /HPV/), liver (Hepatitis B Virus /HBV/, Hepatitis C Virus /HCV/), and stomach cancer (H. pylori) ${ }^{1}$.

Recently, worldwide, an estimated 19.3 million new cancer cases (18.1 million excluding nonmelanoma skin cancer) and almost 10.0 million cancer deaths (9.9 million excluding nonmelanoma skin cancer) occurred in $2020^{2}$. Cancers, due to infectious agents were estimated at 2.2 million in 2018, but exact numbers could not be ascertained due to scarcity of local data, especially in low-SDI countries 3. Hematologic malignancies (HMs) are the fifth most common cancer group in economically developed countries of the world and they originate from the uncontrolled proliferation of hematopoietic and lymphoid cells. These biologically and clinically heterogeneous groups account for $6.5 \%$ of all malignancies around the world, including approximately 9.0\% in the United States and Europe 4,5,. They are traditionally categorized by site according to whether cancer is first detected in the blood (leukemias), lymph nodes (lymphomas - Hodgkin and non-Hodgkin), or bone (myelomas) ${ }^{7}$.

Hodgkin lymphoma (HL), is called Hodgkin's disease (HD), considered as 1 of many types of lymphoma and the most common type of HD is the "classic" (CHD, 95\%) form containing abnormal lymphocytes, known as Reed-Sternberg cells with an estimated annual incidence of 80,000 cases worldwide ${ }^{8,9}$.

Non-Hodgkin Lymphoma (NHL): The development of NHL begins when healthy B cells, T cells, or NK cells in the lymphatic system change and grow out of control, which may form a tumor ${ }^{10,11 .}$

Multiple myeloma (MM): Multiple myeloma is a cancer of plasma cells. In 2016, there were about 130,000 cases of myeloma, translating to an age-standardized incidence rate of 2.1 per 100,000 persons. Multiple myeloma caused 98,437 deaths globally, with an age-standardized incidence ratio of 1.5 per 100,000 persons. That means from 1990 to 2016, incident cases of MM increased by 126\% globally and deaths increased $94 \%{ }^{12}$.

Leukemia (LEU) is the cancer of the body's blood-forming cells, including the bone marrow and the lymphatic system. Several types of leukemia exist. They might develop in children or adults and they can appear as acute, or chronic diseases. Leukemia represents the 11th and 10th most frequent cause of cancer occurrence and death worldwide (13). Risk factors and/or putative causes for hematological malignancies are gender, age, exposure to chemicals (benzene), radiation, congenital syndromes (Fanconi, Dawn, Bloom syndrome, etc), viral infections (Epstein-Barr virus, Human T-cell Leukemia Virus /HTLV/, hepatitis-C, HIV/AIDS, etc.), bacterial infections (H. pylori in MALT lymphoma) ${ }^{14-22}$.

The human microbiome has several beneficial effects in terms of maintaining appropriate human health, but its alteration has been implicated in the development of many illnesses. and gut microbiota dysbiosis - imbalances in the composition and function of these intestinal microbes - is associated with diseases ranging from localized gastroenterology disorders to neurologic, respiratory, metabolic, hepatic, and cardiovascular illnesses. Gut microbial species are being explored in thefield of oncology also. Of specific interest is the capacity of some commensal bacteria to modulate the tumor microenvironment and anticancer therapies ${ }^{23}$. In one of our previous works, associations have been observed between antibiotic consumption patterns and the incidence of major cancers in European countries ${ }^{24}$. Similarly, repeated antibiotic consumption is associated with cancer prevalence, probably acting through the modification of the gut microbiome ${ }^{25}$. Publications indicate the possible role of the different intestinal microbiome in the development of hematological malignancies and even anticancer treatment. The microbiome can influence hematologic malignancies in several ways, including directly through metabolites and toxins, orindirectly via the innate and adaptive immune system ${ }^{26-28}$. 
Concept/hypothesis: Antibiotic consumption patterns in European countries are extremely different. The most preferred antibiotics used in certain countries are narrow-spectrum penicillin and tetracycline, while in others; broad-spectrum antibiotics are most frequently consumed. The calculated average ratio of broad-/narrow-spectrum antibiotics for the years of 2010-2019 (10 years, expressed in Defined Daily Dose/ 1000 inhabitants/ Day /DID/) is the highest in Greek $(321,94)$ and the lowest is in Norway $(0,19)$. Based on this 1694,42 fold difference it could be suspected that those very different antibiotic consumption patterns might influence the composition of the gut flora differently and hence, the altered gut flora (dysbiosis) might promote, or inhibit the development of certain ailments. The incidence of different hematological cancers (HL NHL, MM, LEU, 29-32) estimated for 2020 shows considerable differences between European countries. The highest incidence rate for HD is in Cyprus $(4,69)$, the lowest is in Romania $(1,3)$. NHL incidence is highest in Slovenia $(28,1)$, the lowest is in Bulgaria $(8,7)$. The MM incidence is highest in Iceland $(11,7)$, the lowest is in Bulgaria $(2,2)$. The incidence of LEU is highest in Belgium (21) and the lowest is in Bulgaria $(7,5)$. The difference between the highest and lowest incidence rate of the above hematological malignancies is approximately 3-5 fold ${ }^{29-32}$.

It can be hypothesized that different classes of antibiotics, producing different modifications on the gut flora, might promote or inhibit the development of different hematological malignancies and this activity could be attached to different antibiotic classes. We have hypothesized also that if antibiotics, through different putative mechanisms, published in the scientific literature, could influence the hematological oncogenesis, those antibiotic consumption patterns might be reflected in the incidence of different hematological malignancies in the different countries included in the study.

Materials and methods: Databases were calculated from publicly available antibiotic consumption figures (ECDC yearly reports for 2009-2019 ${ }^{33}$ and the incidence of hematological malignancies (HD, NHD, MM, LEU) estimated for 2020 and featured in the European Cancer Information System (ECIS) for 30 European countries. Average yearly consumption of total systemic antibiotics (ATC classification J01) expressed in Defined Daily Dose/1000 Inhabitants/ Day (DID) was calculated similarly with major antibiotic classes at ATC level 3 and 4 as tetracycline (J01A), penicillin (J01C), broad-spectrum, betalactamase sensitive penicillin (J01CA), narrow spectrum, beta-lactamase sensitive penicillin (J01CE), narrow spectrum, beta-lactamase resistant penicillin (J01CF), broad-spectrum, beta-lactamase resistant combination penicillin (J01CR), cephalosporin (J01D), macrolide and lincosamides, streptogramins (J01F), a quinolone (J01M). The average ratio of broad-/narrow-spectrum (B/N) antibiotic consumption/countries have been calculated also. Antibiotic consumption data and the incidence of hematological malignancies were recorded by countries and featured in a spreadsheet (Table 1.). Diagrams for demonstrating positive and negative associations between certain hematological malignancies and antibiotic consumption data were created (Diagram 1-4). Spearman correlation was applied to estimate the correlation between antibiotic consumption and the prevalence data of hematological malignancies. A significant correlation was considered when $p$ values were $\leq 0.05$. Positive (supportive) and negative (non-supportive) significant correlations were considered and evaluated. Statistical results were recorded and featured in the same table. (Table1.).

The spreadsheet was formulated for comparing the rank order of countries (first ten positions) with the highest incidence of different hematological malignancies and the rank order of consumption of antibiotic classes showing positive ("enhancing") and negative ("inhibiting") correlation with the hematological malignancies in the same countries (Table 2.). 


\begin{tabular}{|c|c|c|c|c|c|c|c|c|c|c|c|c|c|c|c|}
\hline \multirow[t]{2}{*}{ Countries } & \multirow[t]{2}{*}{\begin{tabular}{l} 
Average. \\
total \\
antibiotic \\
consumpti \\
on. \\
$2010-19$ in \\
DID \\
\\
\multicolumn{1}{c}{ J01 }
\end{tabular}} & \multicolumn{10}{|c|}{$\begin{array}{l}\qquad \text { Antibiotic classes } \\
\text { Average consumption (2010-2019) of tetracycline (J01A). penicillin (J01C). beta-lactamase } \\
\text { sensitive broad-spectrum penicillin (J01CA). beta-lactamase sensitive. narrow-spectrum } \\
\text { penicillin (J01CE). beta-lactamase resistant. narrow-spectrum penicillin (J01CF). broad- } \\
\text { spectrum. beta-lactamase resistant combination penicillin (J01CR). cephalosporin (J01D). } \\
\text { macrolide (and lincosamides. streptogramines). quinolone (J01F). quinolone (J01M). ratio of } \\
\text { broad-/narrow spectrum antibiotics expressed in Defined Daily Dose/ } 1000 \text { inhabitants/ day } \\
\text { (DID) }\end{array}$} & \multicolumn{4}{|c|}{$\begin{array}{c}\text { Incidence of } \\
\text { hematological } \\
\text { malignancies } 2020 \\
\text { (100000/cases) }\end{array}$} \\
\hline & & J01A & J01C & J01CA & J01CE & J01CF & J01CR & J01D & J01F & $\mathrm{J} 01 \mathrm{M}$ & $\mathrm{B} / \mathrm{N}$ & $H L$ & NHL & $M M$ & $L E U$ \\
\hline Austria & 12.00 & 0.89 & 4.79 & 0.79 & 0.79 & 0.01 & 3.21 & 1.55 & 2.98 & 1.22 & 6.71 & 1.7 & 15.2 & 6.5 & 14 \\
\hline Belgium & 22.25 & 2.02 & 10.33 & 4.94 & 0.03 & 0.26 & 5.10 & 1.40 & 3.40 & 2.23 & 85.39 & 3.1 & 24.4 & 9.4 & 21 \\
\hline Bulgaria & 17.87 & 1.72 & 5.43 & 3.13 & 0.19 & 0.00 & 2.11 & 3.42 & 3.65 & 2.61 & 27.34 & 1.8 & 8.7 & 2.2 & 7.5 \\
\hline Croatia & 17.32 & 1.11 & 7.64 & 1.97 & 0.66 & 0.00 & 5.00 & 2.84 & 2.88 & 1.44 & 8.21 & 2.3 & 13.2 & 6.4 & 12.1 \\
\hline Cyprus* & 26.64 & 3.38 & 9.14 & 2.43 & 0.08 & 0.02 & 6.62 & 5.47 & 2.92 & 4.75 & 32.43 & 4.6 & 23.2 & 10.2 & 18.9 \\
\hline Czechia & 16.64 & 2.11 & 5.96 & 1.16 & 1.85 & 0.05 & 2.88 & 1.86 & 3.67 & 0.95 & 4.28 & 2.4 & 17.5 & 5.3 & 14.2 \\
\hline Denmark & 15.10 & 1.63 & 9.61 & 3.19 & 4.28 & 1.39 & 0.74 & 0.03 & 1.91 & 0.48 & 0.55 & 2.7 & 25.1 & 8.8 & 15.4 \\
\hline Estonia & 10.25 & 1.58 & 3.31 & 1.72 & 0.19 & 0.00 & 1.40 & 1.10 & 2.36 & 0.83 & 12.38 & 2.5 & 17.7 & 6.7 & 14.7 \\
\hline Finland & 15.73 & 3.89 & 4.78 & 2.65 & 1.24 & 0.04 & 0.85 & 2.13 & 1.13 & 0.76 & 0.59 & 3 & 22.3 & 7 & 11.5 \\
\hline France & 23.54 & 3.19 & 12.37 & 7.34 & 0.17 & 0.23 & 4.64 & 2.03 & 3.27 & 1.63 & 36.71 & 3.1 & 21 & 10 & 16.8 \\
\hline Germany & 12.97 & 2.11 & 3.43 & 2.24 & 0.75 & 0.01 & 0.44 & 2.82 & 2.32 & 1.25 & 5.67 & 2.8 & 19.6 & 7.3 & 14.8 \\
\hline Greece ${ }^{*}$ & 31.19 & 2.49 & 9.56 & 4.42 & 0.08 & 0.00 & 5.07 & 7.61 & 7.50 & 2.63 & 321.94 & 3.2 & 13.4 & 7.5 & 15.4 \\
\hline Hungary & 13.62 & 1.18 & 4.63 & 0.88 & 0.26 & 0.00 & 3.49 & 1.96 & 2.93 & 2.22 & 42.74 & 2 & 15.9 & 4.3 & 13.6 \\
\hline Iceland $^{*}$ & 19.03 & 4.81 & 9.03 & 3.23 & 2.08 & 1.04 & 2.69 & 0.58 & 1.61 & 0.91 & 1.50 & 2.1 & 18.9 & 11.7 & 11.3 \\
\hline Ireland & 19.83 & 2.75 & 9.56 & 2.96 & 1.06 & 1.40 & 4.15 & 1.18 & 4.12 & 0.83 & 4.80 & 3.2 & 23.9 & 9.2 & 16.3 \\
\hline Italy & 21.70 & 0.54 & 9.97 & 2.61 & 0.00 & 0.01 & 7.35 & 2.32 & 4.49 & 3.13 & 165.50 & 3.4 & 20.3 & 8.1 & 13.4 \\
\hline Latvia & 11.14 & 2.28 & 4.29 & 2.89 & 0.05 & 0.00 & 1.35 & 0.55 & 1.72 & 0.99 & 12.46 & 2.7 & 13 & 5.8 & 14.3 \\
\hline Lithuania* $^{*}$ & 13.90 & 1.47 & 6.53 & 4.90 & 0.23 & 0.00 & 1.41 & 1.20 & 1.99 & 0.95 & 9.29 & 2.1 & 17 & 6.5 & 18.3 \\
\hline Luxembourg & 21.89 & 1.81 & 8.60 & 3.13 & 0.02 & 0.18 & 5.29 & 3.35 & 3.90 & 2.50 & 48.00 & 2.5 & 19.3 & 8.7 & 15.2 \\
\hline Malta & 19.26 & 1.33 & 6.41 & 0.49 & 0.10 & 0.06 & 5.77 & 4.24 & 3.92 & 2.30 & 100.25 & 2.6 & 21.4 & 6.2 & 9.7 \\
\hline Netherlands & 9.44 & 2.24 & 3.07 & 1.31 & 0.26 & 0.44 & 1.06 & 0.04 & 1.42 & 0.77 & 8.82 & 2.7 & 23.6 & 8.6 & 13.6 \\
\hline Norway & 15.16 & 2.97 & 6.03 & 2.12 & 3.27 & 0.63 & 0.01 & 0.09 & 1.42 & 0.45 & 0.19 & 2.9 & 21.1 & 11.3 & 15.6 \\
\hline Poland & 20.90 & 2.30 & 6.66 & 3.42 & 0.24 & 0.01 & 2.98 & 2.82 & 4.18 & 1.32 & 28.22 & 1.6 & 11.9 & 6.4 & 12.6 \\
\hline Portugal & 17.65 & 0.86 & 8.44 & 1.70 & 0.02 & 0.53 & 6.19 & 1.59 & 2.99 & 2.14 & 36.51 & 2.3 & 18.7 & 7.7 & 13.7 \\
\hline Romania & 25.76 & 1.08 & 12.23 & 4.63 & 0.80 & 0.66 & 6.14 & 4.83 & 2.91 & 3.33 & 12.11 & 1.3 & 9.7 & 4.5 & 9.9 \\
\hline Slovakia & 19.92 & 1.61 & 5.86 & 1.00 & 1.21 & 0.00 & 3.65 & 4.58 & 5.30 & 1.94 & 9.52 & 2.8 & 14.4 & 8.5 & 16.1 \\
\hline Slovenia & 11.72 & 0.41 & 6.97 & 2.27 & 1.68 & 0.16 & 2.86 & 0.33 & 1.80 & 1.10 & 2.94 & 2.3 & 28.1 & 7.9 & 15 \\
\hline Spain & 19.78 & 1.03 & 10.94 & 4.34 & 0.09 & 0.21 & 6.32 & 1.86 & 2.43 & 2.53 & 54.65 & 2.9 & 16.9 & 6.6 & 12.3 \\
\hline Sweden & 12.25 & 2.74 & 6.18 & 1.07 & 3.38 & 1.50 & 0.22 & 0.14 & 0.59 & 0.68 & 0.20 & 2.1 & 17.7 & 7.8 & 13 \\
\hline UK & 17.18 & 4.70 & 6.55 & 3.51 & 0.82 & 1.44 & 0.78 & 0.32 & 2.93 & 0.45 & 1.44 & 3.5 & 26.2 & 9.9 & 17 \\
\hline \multicolumn{16}{|l|}{ HL } \\
\hline Spearman $r$ & 0.321 & 0.399 & 0.267 & 0.220 & -0.227 & 0.192 & 0.125 & 0.003 & 0.146 & $\begin{array}{l}-0.004 \\
\end{array}$ & 0.137 & & & & \\
\hline Spearman $p$ & 0.084 & 0.029 & 0.154 & 0.243 & 0.227 & 0.311 & 0.510 & 0.987 & 0.441 & 0.985 & 0.469 & & & & \\
\hline \multicolumn{16}{|l|}{ NHL } \\
\hline Spearman $r$ & -0.087 & 0.257 & 0.161 & 0.023 & 0.163 & 0.580 & -0.154 & -0.46 & -0.225 & -0.380 & -0.270 & & & & \\
\hline Spearman $p$ & 0.649 & 0.171 & 0.394 & 0.902 & 0.389 & 0.001 & 0.417 & 0.011 & 0.232 & 0.038 & 0.149 & & & & \\
\hline \multicolumn{16}{|l|}{ MM } \\
\hline Spearman $r$ & 0.223 & 0.492 & 0.366 & 0.231 & 0.120 & 0.574 & -0.070 & -0.34 & -0.126 & -0.272 & -0.222 & & & & \\
\hline Spearman $p$ & 0.236 & $\underline{0.006}$ & $\underline{0.047}$ & 0.220 & 0.526 & $\underline{0.001}$ & 0.715 & 0.063 & 0.506 & 0.146 & 0.238 & & & & \\
\hline \multicolumn{16}{|l|}{ LEU } \\
\hline Spearman $r$ & 0.112 & 0.267 & 0.174 & 0.258 & -0.068 & 0.088 & -0.068 & -0.20 & -0.126 & -0.161 & -0.036 & & & & \\
\hline Spearman $p$ & 0.557 & 0.154 & 0.358 & 0.168 & 0.719 & 0.643 & 0.722 & 0.278 & 0.506 & 0.395 & 0.849 & & & & \\
\hline
\end{tabular}

Table 1. Comparison of average antibiotic consumption (2010-2019) expressed in Defined Daily Dose/ 1000 inhabitants/ day (DID), and the incidence of hematologic malignancies estimated for 2020.

Spearman correlation indicated (bold, italics, underlined) significant positive associations between HL and tetracycline (J01A), NHL and narrow spectrum, beta-lactamase resistant penicillin (J01CF), MM and tetracycline (J01A), and penicillin (J01C) particularly with the narrow spectrum, beta-lactamase resistant penicillin (J01CF). Negative significance was found (bold, italics) between NHL and cephalosporin (J01D) and quinolone (J01M). The tendency for a positive correlation between HD and the total consumption of systemic antibiotics (J01, bold) was observed also. A tendency for negative correlation has been detected between MM and cephalosporin (J01D, bold). No associations of any classes of antibiotics and LEU was estimated. 
Decreasing rank order of "enhancer" antibiotic consumption (tetracycline: J01A, penicillin group: (J01C), narrow spectrum, penicillinase-

Decreasing rank order of "enhancer" antibiotic consumption (tetracycline: J01A, penicillin group: (J01C), narrow spectrum, penicillinaseresistant penicillin :J01CF) in European countries (average consumption for 2010-2019 in DID), compared to the decreasing rank orders of the incidence of the major groups of hematological malignancies 2020 (HL, NHL, MM, LEU)

\begin{tabular}{|c|c|c|c|c|c|c|c|c|c|c|c|c|c|c|c|}
\hline Countries & J01A & Countries & $H L$ & Countries & J01A & Countries & NHL & Countries & J01A & Countries & $M M$ & Countries & J01A & Countries & LEU \\
\hline Iceland & 4,81 & Cyprus & $\underline{4,6}$ & Iceland & 4,81 & Slovenia & 28,1 & Iceland & $\underline{\underline{4}, 81}$ & Iceland & $\underline{11,7}$ & Iceland & $\underline{4,81}$ & Belgium & 21 \\
\hline$\underline{\underline{U K}}$ & $\underline{4,7}$ & $\underline{\underline{U K}}$ & $\underline{3,5}$ & $\underline{U K}$ & $\underline{4,7}$ & $\underline{\underline{U K}}$ & $\underline{26,2}$ & $\underline{\underline{U K}}$ & $\underline{4,7}$ & Norway & $\underline{11,3}$ & $\underline{U K}$ & $\underline{4,7}$ & Cyprus & $\underline{18,9}$ \\
\hline Finland & $\underline{3,89}$ & Italy & 3,4 & Finland & $\underline{3,89}$ & Denmark & 25,1 & Finland & 3,89 & Cyprus & $\underline{10,2}$ & Finland & 3,89 & Lithuania & 18,3 \\
\hline Cyprus & 3,38 & Greece & $\underline{3,2}$ & Cyprus & $\underline{3,38}$ & Belgium & 24,4 & Cyprus & $\underline{3,38}$ & France & $\underline{10}$ & Cyprus & $\underline{3,38}$ & $\underline{U K}$ & $\underline{17}$ \\
\hline France & $\underline{3,19}$ & Ireland & $\underline{3,2}$ & France & 3,19 & Ireland & $\underline{23,9}$ & France & $\underline{3,19}$ & $\underline{U K}$ & $\underline{9,9}$ & France & $\underline{3,19}$ & France & $\underline{16,8}$ \\
\hline Norway & $\underline{2,97}$ & Belgium & 3,1 & Norway & $\underline{2,97}$ & Netherlands & 23,6 & Norway & $\underline{2,97}$ & Belgium & 9,4 & Norway & $\underline{2,97}$ & Ireland & $\underline{16,3}$ \\
\hline Ireland & $\underline{2,75}$ & France & $\underline{3,1}$ & Ireland & $\underline{2,75}$ & Cyprus & $\underline{23,2}$ & Ireland & $\underline{2,75}$ & Ireland & $\underline{9,2}$ & Ireland & 2,75 & Slovakia & 16,1 \\
\hline Sweden & 2,74 & Finland & $\underline{3}$ & Sweden & 2,74 & Finland & $\underline{22,3}$ & Sweden & 2,74 & Denmark & 8,8 & Sweden & 2,74 & Norway & $\underline{15,6}$ \\
\hline Greece & 2,49 & Norway & 2,9 & Greece & 2,49 & Malta & 21,4 & Greece & 2,49 & Luxembourg & 8,7 & Greece & 2,49 & Denmark & 15,4 \\
\hline Poland & 2,3 & Spain & 2,9 & Poland & 2,3 & Norway & $\underline{21,1}$ & Poland & 2,3 & Netherlands & 8,6 & Poland & 2,3 & Greece & $\underline{15,4}$ \\
\hline Countries & J01CF & Countries & HL & Countries & J01CF & Countries & NHL & Countries & J01CF & Countries & $M M$ & Countries & J01CF & Countries & LEU \\
\hline Sweden & 1,5 & Cyprus & 4,6 & Sweden & 1,5 & Slovenia & 28,1 & Sweden & 1,5 & Iceland & $\underline{11,7}$ & Sweden & 1,5 & Belgium & $\underline{21}$ \\
\hline$\underline{U K}$ & $\underline{1,44}$ & $\underline{U K}$ & $\underline{3,5}$ & $\underline{U K}$ & 1,44 & $\underline{U K}$ & $\underline{26,2}$ & $\underline{U K}$ & $\underline{1,44}$ & Norway & $\underline{11,3}$ & $\underline{U K}$ & $\underline{1,44}$ & Cyprus & 18,9 \\
\hline Ireland & $\underline{1, \underline{4}}$ & Italy & 3,4 & Ireland & $\underline{1, \underline{4}}$ & Denmark & $\underline{25,1}$ & Ireland & $\underline{1, \underline{4}}$ & Cyprus & 10,2 & Ireland & $\underline{1, \underline{4}}$ & Lithuania & 18,3 \\
\hline Denmark & 1,39 & Greece & 3,2 & Denmark & $\underline{1,39}$ & Belgium & $\underline{24,4}$ & Denmark & $\underline{1,39}$ & France & 10 & Denmark & $\underline{1,39}$ & $\underline{U K}$ & $\underline{17}$ \\
\hline Iceland & 1,04 & Ireland & 3,2 & Iceland & 1,04 & Ireland & 23,9 & Iceland & 1,04 & $\underline{\underline{U K}}$ & $\underline{9,9}$ & Iceland & 1,04 & France & 16,8 \\
\hline Romania & 0,66 & Belgium & $\underline{3,1}$ & Romania & 0,66 & Netherlands & $\underline{23,6}$ & Romania & 0,66 & Belgium & $\underline{9,4}$ & Romania & 0,66 & Ireland & $\underline{16, \underline{3}}$ \\
\hline Norway & $\underline{0,63}$ & France & 3,1 & Norway & $\underline{0,63}$ & Cyprus & 23,2 & Norway & $\underline{0,63}$ & Ireland & $\underline{9,2}$ & Norway & $\underline{0,63}$ & Slovakia & 16,1 \\
\hline Portugal & 0,53 & Finland & 3 & Portugal & 0,53 & Finland & 22,3 & Portugal & 0,53 & Denmark & $\underline{8,8}$ & Portugal & 0,53 & Norway & $\underline{15,6}$ \\
\hline Netherlands & 0,44 & Norway & $\underline{2,9}$ & Netherlands & $\underline{0,44}$ & Malta & 21,4 & Netherlands & $\underline{0,44}$ & Luxembourg & 8,7 & Netherlands & 0,44 & Denmark & $\underline{15,4}$ \\
\hline Belgium & $\underline{0,26}$ & Spain & 2,9 & Belgium & $\underline{0,26}$ & Norway & $\underline{21,1}$ & Belgium & $\underline{0,26}$ & Netherlands & $\underline{8,6}$ & Belgium & $\underline{0,26}$ & Greece & 15,4 \\
\hline Countries & J01C & Countries & 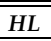 & Countries & J01C & Countries & NHL & Countries & J01C & Countries & 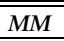 & Countries & J01C & Countries & 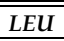 \\
\hline France & $\underline{12,37}$ & Cyprus & $\underline{4,6}$ & France & 12,37 & Slovenia & 28,1 & France & $\underline{12,37}$ & Iceland & $\underline{11,7}$ & France & $\underline{12,37}$ & Belgium & $\underline{21}$ \\
\hline Romania & 12,23 & UK & 3,5 & Romania & 12,23 & UK & 26,2 & Romania & 12,23 & Norway & 11,3 & Romania & 12,23 & Cyprus & $\underline{18,9}$ \\
\hline Spain & 10,94 & Italy & $\underline{3,4}$ & Spain & 10,94 & Denmark & $\underline{25,1}$ & Spain & 10,94 & Cyprus & $\underline{10,2}$ & Spain & 10,94 & Lithuania & 18,3 \\
\hline Belgium & 10,33 & Greece & $\underline{3,2}$ & Belgium & $\underline{10,33}$ & Belgium & $\underline{24,4}$ & Belgium & $\underline{10,33}$ & France & $\underline{10}$ & Belgium & $\underline{10,33}$ & UK & 17 \\
\hline Italy & $\underline{9,97}$ & Ireland & $\underline{3,2}$ & Italy & 9,97 & Ireland & $\underline{23,9}$ & Italy & 9,97 & UK & 9,9 & Italy & 9,97 & France & $\underline{16,8}$ \\
\hline Denmark & 9,61 & Belgium & $\underline{3,1}$ & Denmark & $\underline{9,61}$ & Netherlands & 23,6 & Denmark & $\underline{9,61}$ & Belgium & $\underline{9,4}$ & Denmark & $\underline{9,61}$ & Ireland & $\underline{16, \underline{3}}$ \\
\hline Greece & $\underline{9,56}$ & France & 3,1 & Greece & 9,56 & Cyprus & $\underline{23,2}$ & Greece & 9,56 & Ireland & 9,2 & Greece & 9,56 & Slovakia & 16,1 \\
\hline Ireland & $\underline{9,56}$ & Finland & 3 & Ireland & $\underline{9,56}$ & Finland & 22,3 & Ireland & $\underline{9,56}$ & Denmark & $\underline{8,8}$ & Ireland & $\underline{9,56}$ & Norway & 15,6 \\
\hline Cyprus & $\underline{9,14}$ & Norway & 2,9 & Cyprus & $\underline{9,14}$ & Malta & 21,4 & Cyprus & $\underline{9,14}$ & Luxembourg & 8,7 & Cyprus & $\underline{9,14}$ & Denmark & $\underline{15,4}$ \\
\hline Iceland & 9,03 & Spain & $\underline{2,9}$ & Iceland & 9,03 & Norway & 21,1 & Iceland & $\underline{9,03}$ & Netherlands & 8,6 & Iceland & 9,03 & Greece & 15,4 \\
\hline
\end{tabular}

Increasing (inverse) rank order of the average consumption (2010-2019, in DID) of "protective" (inhibitory) antibiotics (cephalosporin: J01D, quinolone: J01M), compared to the reducing rank order of the groups of hematological malignancies (incidence of HL, NHL, MM, LEU 2020).

\begin{tabular}{|c|c|c|c|c|c|c|c|c|c|c|c|c|c|c|c|}
\hline Countries & J01D & Countries & $H L$ & Countries & J01D & Countries & NHL & Countries & J01D & Countries & $M M$ & Countries & J01D & Countries & LEU \\
\hline Denmark & 0,03 & Cyprus & 4,6 & Denmark & $\underline{0,03}$ & Slovenia & $\underline{28,1}$ & Denmark & $\underline{0,03}$ & Iceland & $\underline{11,7}$ & Denmark & $\underline{0,03}$ & Belgium & 21 \\
\hline Netherlands & 0,04 & $\underline{\underline{U K}}$ & $\underline{3,5}$ & Netherlands & $\underline{0,04}$ & $\underline{\underline{U K}}$ & $\underline{26,2}$ & Netherlands & $\underline{0,04}$ & Norway & $\underline{11, \underline{3}}$ & Netherlands & 0,04 & Cyprus & 18,9 \\
\hline Norway & $\underline{0,09}$ & Italy & 3,4 & Norway & $\underline{0,09}$ & Denmark & $\underline{25,1}$ & Norway & $\underline{0,09}$ & Cyprus & 10,2 & Norway & $\underline{0,09}$ & Lithuania & 18,3 \\
\hline Sweden & 0,14 & Greece & 3,2 & Sweden & 0,14 & Belgium & 24,4 & Sweden & 0,14 & France & 10 & Sweden & 0,14 & $\underline{\underline{U K}}$ & $\underline{17}$ \\
\hline$\underline{\underline{U K}}$ & $\underline{0,32}$ & Ireland & $\underline{3,2}$ & $\underline{U K}$ & $\underline{0,32}$ & Ireland & $\underline{23,9}$ & $\underline{U K}$ & $\underline{0,32}$ & $\underline{\underline{U K}}$ & $\underline{9,9}$ & $\underline{\underline{U K}}$ & $\underline{0,32}$ & France & 16,8 \\
\hline Slovenia & 0,33 & Belgium & 3,1 & Slovenia & $\underline{0,33}$ & Netherlands & $\underline{23,6}$ & Slovenia & 0,33 & Belgium & 9,4 & Slovenia & 0,33 & Ireland & $\underline{16,3}$ \\
\hline Latvia & 0,55 & France & 3,1 & Latvia & 0,55 & Cyprus & 23,2 & Latvia & 0,55 & Ireland & $\underline{9,2}$ & Latvia & 0,55 & Slovakia & 16,1 \\
\hline Iceland & 0,58 & Finland & 3 & Iceland & 0,58 & Finland & 22,3 & Iceland & $\underline{0,58}$ & Denmark & $\underline{8,8}$ & Iceland & 0,58 & Norway & $\underline{15,6}$ \\
\hline Estonia & 1,1 & Norway & $\underline{2,9}$ & Estonia & 1,1 & Malta & 21,4 & Estonia & 1,1 & Luxembourg & 8,7 & Estonia & 1,1 & Denmark & $\underline{15,4}$ \\
\hline Ireland & $\underline{1,18}$ & Spain & 2,9 & Ireland & $\underline{\underline{1,18}}$ & Norway & $\underline{21,1}$ & $\underline{\text { Ireland }}$ & $\underline{\underline{1,18}}$ & Netherlands & $\underline{8,6}$ & Ireland & $\underline{1,18}$ & Greece & 15,4 \\
\hline Countries & $\mathrm{J} 01 \mathrm{M}$ & Countries & $H L$ & Countries & J01M & Countries & NHL & Countries & J01M & Countries & $M M$ & Countries & $\mathrm{J} 01 \mathrm{M}$ & Countries & LEU \\
\hline Norway & $\underline{0,45}$ & Cyprus & 4,6 & Norway & $\underline{0,45}$ & Slovenia & 28,1 & Norway & $\underline{0,45}$ & Iceland & $\underline{11,7}$ & Norway & $\underline{0,45}$ & Belgium & 21 \\
\hline$\underline{\underline{U K}}$ & $\underline{0,45}$ & $\underline{\underline{U K}}$ & $\underline{3,5}$ & $\underline{\underline{U K K}}$ & $\underline{0,45}$ & $\underline{\underline{U K}}$ & $\underline{26,2}$ & $\underline{\underline{U K}}$ & $\underline{0,45}$ & Norway & $\underline{11,3}$ & $\underline{\underline{U K}}$ & $\underline{0,45}$ & Cyprus & 18,9 \\
\hline Denmark & 0,48 & Italy & 3,4 & Denmark & $\underline{0,48}$ & Denmark & $\underline{25,1}$ & Denmark & $\underline{0,48}$ & Cyprus & 10,2 & Denmark & $\underline{0,48}$ & Lithuania & 18,3 \\
\hline Sweden & 0,68 & Greece & 3,2 & Sweden & 0,68 & Belgium & 24,4 & Sweden & 0,68 & France & 10 & Sweden & 0,68 & $\underline{\underline{U K}}$ & $\underline{17}$ \\
\hline Finland & $\underline{0,76}$ & Ireland & $\underline{3,2}$ & Finland & $\underline{0,76}$ & Ireland & $\underline{23,9}$ & Finland & 0,76 & $\underline{\underline{U K}}$ & $\underline{9,9}$ & Finland & 0,76 & France & 16,8 \\
\hline Netherlands & 0,77 & Belgium & 3,1 & Netherlands & $\underline{0,77}$ & Netherlands & $\underline{23,6}$ & Netherlands & $\underline{0,77}$ & Belgium & 9,4 & Netherlands & 0,77 & Ireland & $\underline{16,3}$ \\
\hline Estonia & 0,83 & France & 3,1 & Estonia & 0,83 & Cyprus & 23,2 & Estonia & 0,83 & Ireland & $\underline{9,2}$ & Estonia & 0,83 & Slovakia & 16,1 \\
\hline Ireland & $\underline{0,83}$ & Finland & $\underline{3}$ & Ireland & $\underline{0,83}$ & Finland & $\underline{22,3}$ & Ireland & $\underline{0,83}$ & Denmark & $\underline{8,8}$ & $\underline{\text { Ireland }}$ & $\underline{0,83}$ & Norway & $\underline{15,6}$ \\
\hline Iceland & 0,91 & Norway & $\underline{2,9}$ & Iceland & 0,91 & Malta & 21,4 & Iceland & $\underline{0,91}$ & Luxembourg & 8,7 & Iceland & 0,91 & Denmark & $\underline{15,4}$ \\
\hline Czechia & 0,95 & Spain & 2,9 & Czechia & 0,95 & Norway & $\underline{21,1}$ & Czechia & 0,95 & Netherlands & $\underline{8,6}$ & Czechia & 0,95 & Greece & 15,4 \\
\hline
\end{tabular}

Table 2. Decreasing rank order of "enhancer" antibiotic consumption (J01A, J01CF, J01C) compared to the decreasing rank order of hematological malignancies (HL, NHL, MM, LEU) by countries (first ten positions). A higher overlap between the rank orders is possible indicating that the higher consumption 
of those antibiotics is associated with the higher incidence rate of hematological malignancies in the given countries. Similarly, the less consumption of the "inhibitory" antibiotics (J01D, J01M) seems to be associated with the higher incidence rate of hematological malignancies. Identical countries were written in bold, italics, and underlined. Concordance was considered, when six, out of the ten countries were identified in the rank order of hematological malignancies and antibiotic consumption.

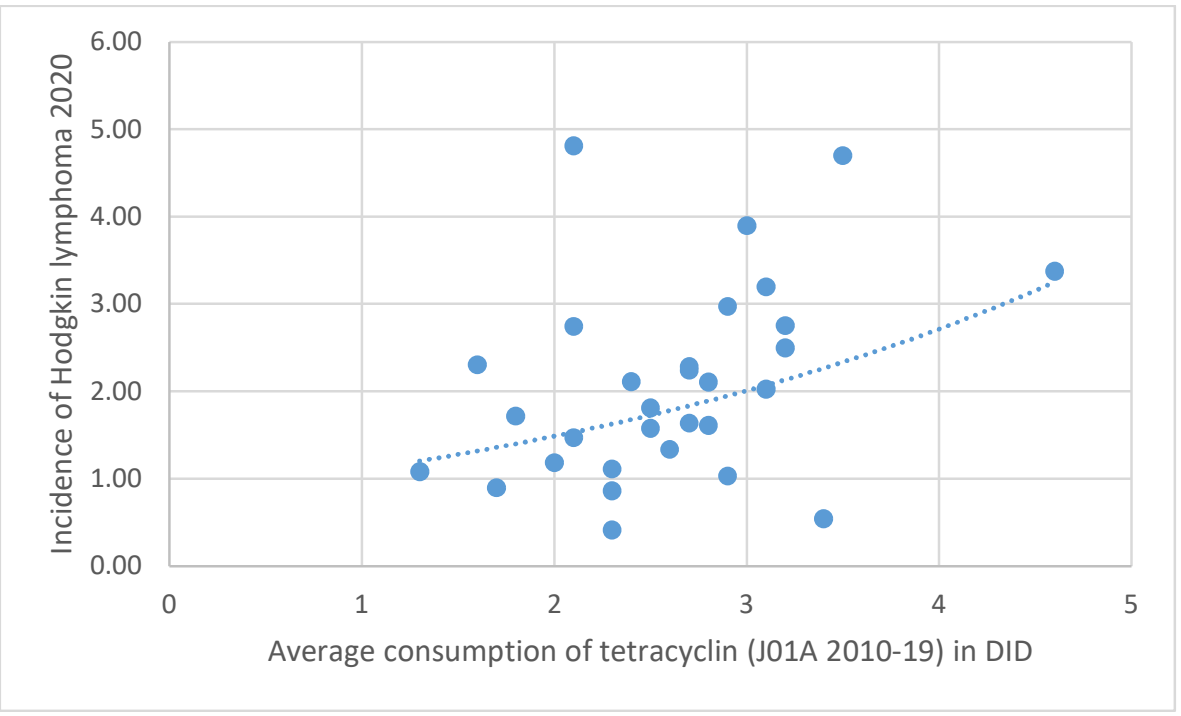

Diagram 1. A significant positive association has been found between tetracycline consumption and the incidence of HD (2020)

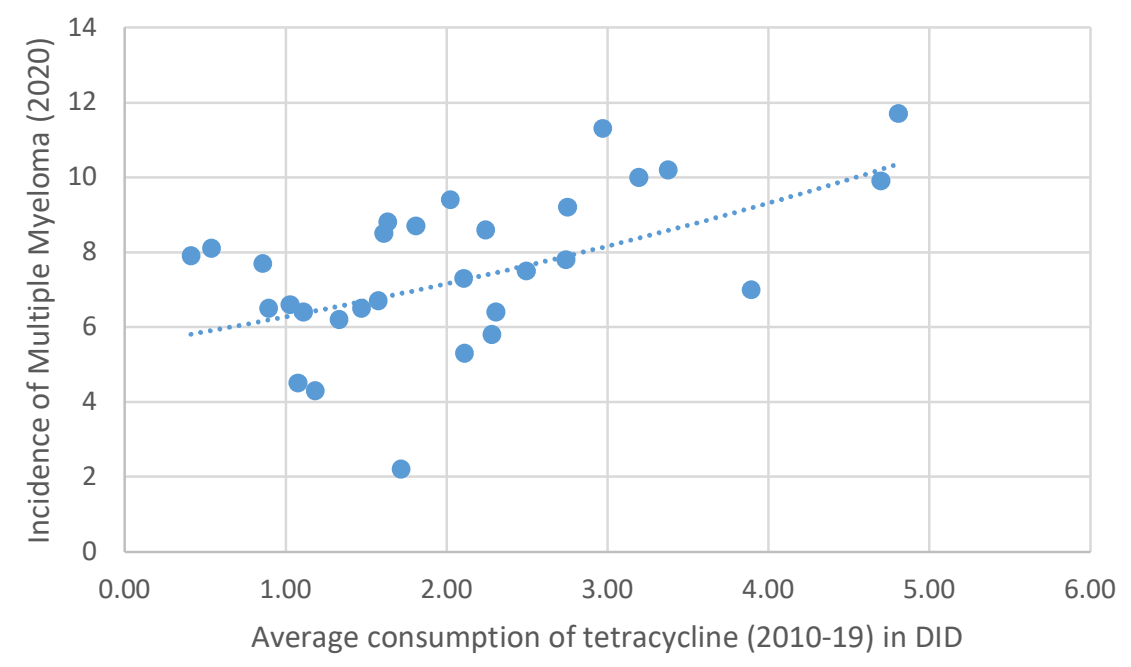

Diagram 2. A significant positive association was seen between tetracycline consumption and the incidence of MM (2020) 


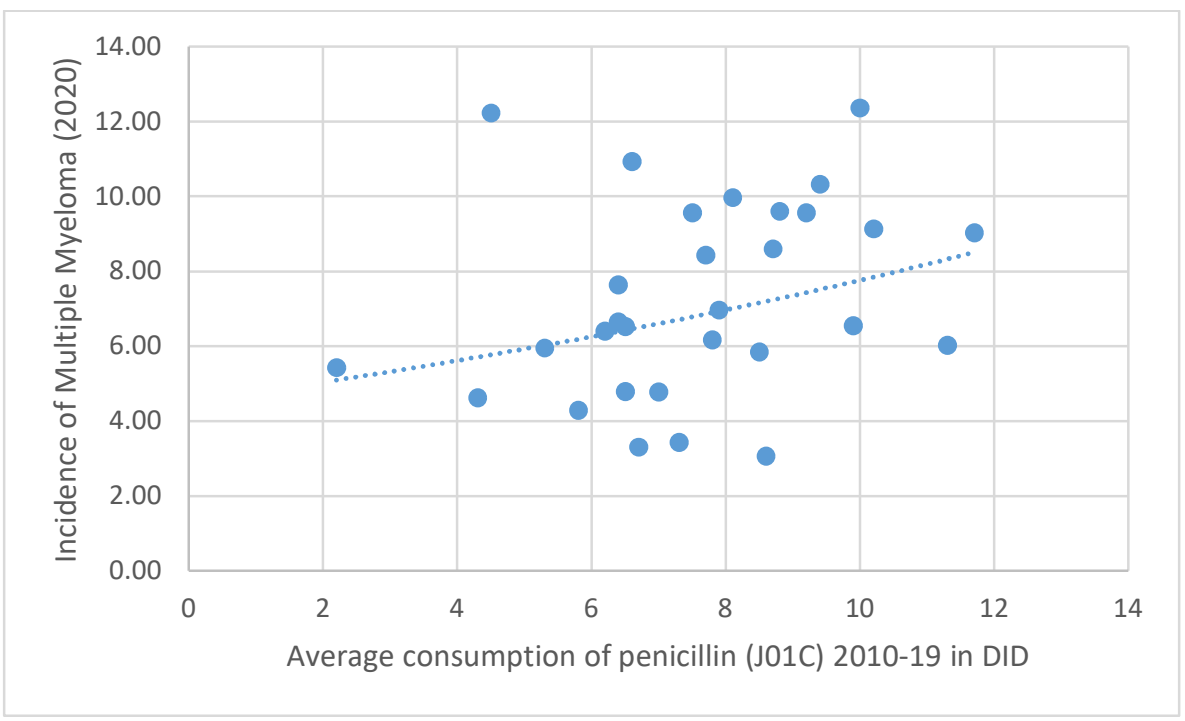

Diagram 3. A significant positive association was found between penicillin consumption and the incidence of MM (2020)

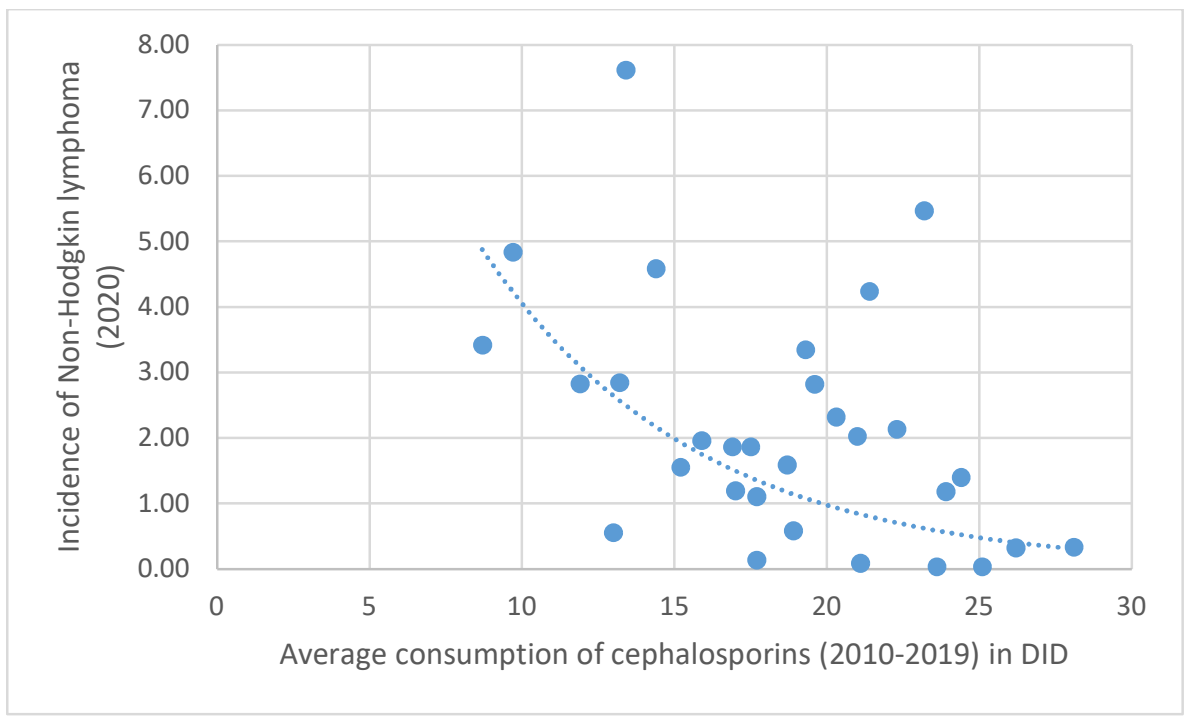

Diagram 4. A significant negative association found between cephalosporin consumption and the incidence of NHL (2020)

Results: The incidence of HL (estimated for 2020) showed strong positive association with the consumption of tetracycline (J01A) according to Spearman calculation $(\boldsymbol{r}=\mathbf{0 . 3 9 9}, \boldsymbol{p}=\mathbf{0 . 0 2 9})$. A similar tendency for positive correlation was observed between HL incidence and the total consumption (J01) of antibiotics for systemic use $(\boldsymbol{r}=\mathbf{0 . 3 2 1}, \boldsymbol{p}=\mathbf{0 . 0 8 4})$. Positive significance was found between the consumption of narrow spectrum, beta-lactamase resistant penicillin (J01CF), and the incidence of NHL $(\boldsymbol{r}=\mathbf{0 . 5 8 0}, \boldsymbol{p}=\mathbf{0 . 0 0 1})$, while a strong negative association was found between cephalosporin consumption (J01C) with the incidence of NHL $(r=\mathbf{0 . 4 6 0}, p=\mathbf{0 . 0 1 1})$ and the quinolone (J01M) with NHL $(r=0.380$, $p=0,038$ ) indicating the possible enhancing effect of tetracycline and the inhibitory effect of cephalosporin and quinolone in the development of NHL. 
The incidence of MM demonstrated positive associations with the consumption of tetracycline (J01A) $(r$ $=0.492, p=0.006)$, with penicillin (J01C) $(r=0.366, p=0.047)$, and narrow spectrum, beta-lactamase resistant penicillin (J01CF), $(\boldsymbol{r}=\mathbf{0 . 5 7 4}, \boldsymbol{p}=\mathbf{0 . 0 0 1})$. Tendency of negative correlation appeared with cephalosporin, similarly to NHL $(r=-0.34, p=0.063)$.

No, associations were observed with LEU and any classes of antibiotics.

Comparing rank orders (first ten positions) of different hematological malignancies with the highest consumption rank order of "enhancer" antibiotics (J01A, J01C, J01CF), we have identified six countries identical with the rank order of HL, MM, and J01A (tetracycline). Six countries were identified in the NHL group and J01CF (narrow spectrum, beta-lactamase resistant penicillin), and seven countries were identified in the MM rank order with the J01CF class of antibiotics. Seven countries, out of ten, were identical with the HL rank order and the highest consumption of the penicillin group (J01C) and six with the NHL rank order. This concordance supports the possible associations between the consumption of different antibiotic classes and certain hematological malignancies.

Similarly, the lowest consumption of "inhibitor" antibiotics is in concordance with the higher incidence of different hematological malignancies. Six countries with the lowest consumption of cephalosporin (J01D) group of antibiotics are identical with the highest incidence (first ten positions) of the rank order of NHL and six are identical with the consumption of quinolone and the NHL.

Discussion: The most densely populated microbial ecosystem that colonizes the human body is found in the gut and is commonly referred to as gut microbiota. It might be considered that gut microbiota is a separate organ itself, and the latest study sets an estimation of over 40 trillion intestinal microorganisms, bringing the ratio closer to 1:1 to somatic cells, expected to be around 30 trillion. The bacteria that comprise the mammal gut microbiota belong primarily to four phyla: Firmicutes, Bacteroidetes, Proteobacteria, and Actinobacteria. Altogether, these phyla account for over $95 \%$ of the total bacteria in the mammalian microbiota. The mean total mucosal surface of the digestive tract can be estimated as @32 $\mathrm{m}^{2}$, of which about $2 \mathrm{~m}^{2}$ refers to the large intestine ${ }^{34}$. The colonization of the intestinal lumen begins at birth and the composition of the gut microbiota is being influenced by several host and external factors and plays a crucial role in maintaining intestinal homeostasis, plays role in the maturation and education of the human immune system, protecting against the colonization of pathogen bacteria, responsible for energy harvest, production of nutrients and vitamins, metabolism of xenobiotics and procarcinogens. One of the most important scientific discoveries of recent years was the disclosure that the intestinal microflora takes part in the bidirectional communication between the gut and the brain. Scientists suggest that human gut microflora may even act as the "second brain" 35 .

Advances in culture-independent research techniques have led to an increased understanding of gut microbiota and the role it plays in health and disease. Several studies indicate the implication of altered microbiome (dysbiosis) in different metabolic disorders (diabetes, obesity) ${ }^{36-38}$, inflammatory bowel disease ${ }^{39-41}$ autism ${ }^{42-44}$, and neurodegenerative diseases Parkinson's disease ${ }^{45-48}$, Alzheimer disease ${ }^{49,50}$ multiple sclerosis ${ }^{51-53}$. Recent scientific advances have significantly contributed to our understanding of the complex connection between the microbiome and cancer, solid tumors, and hematological malignancies alike ${ }^{54-57}$. As it appears in the literature, microorganisms and microbial elements such as lipopolysaccharides (LPS) can up-regulate Toll-like Receptors (TLR)s, which can provoke activation of nuclear factor-kB (NF-kB), which is critical for controlling tumor-associated inflammation ${ }^{58,59}$, invasion, growth, survival, and immunosuppression ${ }^{60}$. Bacterial lipopolysaccharide (LPS) has also been demonstrated to hasten cell proliferation by c-Jun N-terminal Kinase activation ${ }^{61}$. According to reports, different hematological malignancies might show some association between the alterations of gut flora. It was possible to differentiate between the leukemia subjects and the controls based on their microbiota composition. The principal taxa comprise Roseburia, Ruminococcus, Anaerostipes, and Coprococcus with moderately higher abundance in the controls ${ }^{62}$. In a small "twin studies" difference was identified between the microbiome of the survivors of $\mathrm{HL}$, and their unaffected co-twin controls, as it appears to 
have a deficit of rare gut microbes ${ }^{63}$. The microbiota affects hematopoiesis and influences the efficacies of chemotherapy and antimicrobial treatments ${ }^{64}$.

The most extensively used gut microbial flora disruptors are antibiotics, and hence, it can be easily suspected that antibiotics might play a role in producing dysbiosis and contributing to the development of consecutive, non-infectious diseases. On talking about antibiotics producing dysbiosis, we must assume that different classes of antibiotics with different actions on certain microbial taxes might be diverse, as antibiotic sensitivity of gut flora microbiomes is different ${ }^{65}$.

Conclusion: Scientific publications, cited in the References, clearly describe the role of the microbiome in the development of different hematological malignancies. Our study raises the possibility that different antibiotics, by influencing the composition of gut flora (microbiome), might influence the oncogenic process through the gut-brain axis, and through other molecular pathways, which might enhance or inhibit the development of different hematological malignancies. Tetracycline /J01A/, penicillin /J01C/ and particularly narrow spectrum, beta-lactamase resistant penicillin /J01CF/ appears to be promoting the development of certain hematological malignancies, while other groups of antibiotics might inhibit the oncogenic process (cephalosporin, J01D) through the modification of gut flora. The higher consumption rate of "enhancer" and the lower consumption of "inhibitor" antibiotics appears to be associated with the higher incidence of hematological malignancies as it is featured in the comparison of the rank order of hematological malignancies and antibiotic consumption (Table 2).

Strength of the paper: Comparing large, publicly available databases of the incidence of hematological malignancies (HL, NHL, MM, LEU) and the average yearly antibiotic consumptions (2009-2019) published in the ECDC yearly reports from 33 European Countries, indicates the possible role of certain antibiotic classes (tetracycline $/ \mathrm{J} 01 \mathrm{~A} /$, penicillin $/ \mathrm{J} 01 \mathrm{C} /$, narrow spectrum, beta-lactamase resistant penicillin $/ \mathrm{J} 01 \mathrm{CF} /$ ) in the development of some hematological malignancies (HL, NHL, MM). Cephalosporin consumption appears to reduce the risk of those malignancies. This effect can appear through the modification of gut flora.

Weaknesses: This survey could not demonstrate the above association at the individual level, showing the direct effect of antibiotics and the development of hematological malignancies.

\section{ACKNOWLEDGEMENTS}

The researchers received no external funding.

CONFLICTS OF INTEREST:

The authors have no conflict of interest to report.

\section{References:}

1. Global Burden of Disease Cancer Collaboration, Fitzmaurice C, Akinyemiju TF, Al Lami FH, Alam T, Alizadeh-Navaei R, Allen C, Alsharif U, Alvis-Guzman N, Amini E, et al: Global, Regional, and National Cancer Incidence, Mortality, Years of Life Lost, Years Lived With Disability, and Disability-Adjusted LifeYears for 29 Cancer Groups, 1990 to 2016: A Systematic Analysis for the Global Burden of Disease Study. JAMA Oncol. 2018 Nov 1;4(11):1553-1568. doi: 10.1001/jamaoncol.2018.2706. PMID: 29860482; PMCID: PMC6248091

2. Sung H, Ferlay J, Siegel RL, Laversanne M, Soerjomataram I, Jemal A, Bray F. Global Cancer Statistics 2020: GLOBOCAN Estimates of Incidence and Mortality Worldwide for 36 Cancers in 185 Countries. CA Cancer J Clin. 2021 May;71(3):209-249. doi: 10.3322/caac.21660. Epub 2021 Feb 4. PMID: 33538338.

3. de Martel C, Georges D, Bray F, Ferlay J, Clifford GM. Global burden of cancer attributable to infections in 2018: a worldwide incidence analysis. Lancet Glob Health. 2020 Feb;8(2):e180-e190. doi: 10.1016/S2214109X(19)30488-7. Epub 2019 Dec 17. PMID: 31862245.

4. Dighiero G, Hamblin TJ. Chronic lymphocytic leukemia. Lancet. 2008 Mar 22;371(9617):1017-29. doi: 10.1016/S0140-6736(08)60456-0. PMID: 18358929. 
5. Hossain MS, Iqbal MS, Khan MA, Rabbani MG, Khatun H, Munira S, Miah MM, et al Diagnosed hematological malignancies in Bangladesh - a retrospective analysis of over 5000 cases from 10 specialized hospitals. BMC Cancer. 2014 Jun 14;14:438. doi: 10.1186/1471-2407-14-438. PMID: 24929433; PMCID: PMC4063230

6. Uribe-Herranz M, Klein-González N, Rodríguez-Lobato LG, Juan M, de Larrea CF. Gut Microbiota Influence in Hematological Malignancies: From Genesis to Cure. Int J Mol Sci. 2021 Jan 20;22(3):1026. doi: 10.3390/ijms22031026. PMID: 33498529; PMCID: PMC7864170.

7. HMRN - Hematological malignancies

8. https://www.cancer.net/cancer-types/lymphoma-hodgkin/introduction

9. Hjalgrim H., Jarrett R.F. (2020) Epidemiology of Hodgkin Lymphoma. In: Engert A., Younes A. (eds) Hodgkin Lymphoma. Hematologic Malignancies. Springer, Cham. https://doi.org/10.1007/978-3-030$\underline{32482-7 \quad 1}$

10. https://www.cancer.net/cancer-types/lymphoma-non-hodgkin/introduction

11. Chihara D, Nastoupil LJ, Williams JN, Lee P, Koff JL, Flowers CR. New insights into the epidemiology of non-Hodgkin lymphoma and implications for therapy. Expert Rev Anticancer Ther. 2015 May;15(5):531-44. doi: 10.1586/14737140.2015.1023712. Epub 2015 Apr 11. PMID: 25864967; PMCID: PMC4698971

12. https://www.cancernetwork.com/view/multiple-myeloma-incidence-increasing-worldwide-especially-us

13. Miranda-Filho A, Piñeros M, Ferlay J, Soerjomataram I, Monnereau A, Bray F. Epidemiological patterns of leukaemia in 184 countries: a population-based study. Lancet Hematol. 2018 Jan;5(1):e14-e24. doi: 10.1016/S2352-3026(17)30232-6. PMID: 29304322.

14. Jin MW, Xu SM, An Q, Wang P. A review of risk factors for childhood leukemia. Eur Rev Med Pharmacol Sci. 2016 Sep;20(18):3760-3764. PMID: 27735044.

15. https://www.cancer.net/cancer-types/lymphoma-hodgkin/risk-factors,

16. https://www.cancercenter.com/cancer-types/leukemia/risk-factor,

17. https://www.cancer.net/cancer-types/multiple-myeloma/risk-factors

18. https://www.cancer.net/cancer-types/lymphoma-non-hodgkin/risk-factors

19. Pearce L. Non-Hodgkin's lymphoma. Nurs Stand. 2016 Sep 14;31(3):15. doi: 10.7748/ns.31.3.15.s16. PMID: 27745040.

20. Melenotte C, Mezouar S, Mège JL, Gorvel JP, Kroemer G, Raoult D. Bacterial infection and non-Hodgkin's lymphoma. Crit Rev Microbiol. 2020 May;46(3):270-287. doi: 10.1080/1040841X.2020.1760786. Epub 2020 May 15. PMID: 32412856.

21. Rajkumar SV. Multiple myeloma: 2020 update on diagnosis, risk-stratification and management. Am J Hematol. 2020 May;95(5):548-567. doi: 10.1002/ajh.25791. Erratum in: Am J Hematol. 2020 Nov;95(11):1444. PMID: 32212178.

22. Murray PG, Young LS. An etiological role for the Epstein-Barr virus in the pathogenesis of classical Hodgkin lymphoma. Blood. 2019 Aug 15;134(7):591-596. doi: 10.1182/blood.2019000568. Epub 2019 Jun 11. PMID: 31186275.

23. Lynch SV, Pedersen O. The Human Intestinal Microbiome in Health and Disease. N Engl J Med. 2016 Dec 15;375(24):2369-2379. doi: 10.1056/NEJMra1600266. PMID: 27974040.

24. Ternák G, Berényi K, Sümegi A, Szenczi Á, Fodor B, Németh B, Kiss I. Antibiotic Consumption Patterns in European Countries May Be Associated with the Incidence of Major Carcinomas. Antibiotics (Basel). 2020 Sep 25;9(10):643. doi: 10.3390/antibiotics9100643. PMID: 32993020; PMCID: PMC7601152.

25. Boursi B, Mamtani R, Haynes K, Yang YX. Recurrent antibiotic exposure may promote cancer formation-Another step in understanding the role of the human microbiota? Eur J Cancer. 2015 Nov;51(17):2655-64. doi: 10.1016/j.ejca.2015.08.015. Epub 2015 Aug 31. PMID: 26338196; PMCID: PMC4663115

26. Allegra A, Innao V, Allegra AG, Ettari R, Pugliese M, Pulvirenti N, Musolino C. Role of the microbiota in hematologic malignancies. Neth J Med. 2019 Feb;77(2):67-80. PMID: 30895929.

27. Goodman B, Gardner H. The microbiome and cancer. J Pathol. 2018 Apr;244(5):667-676. doi: 10.1002/path.5047. Epub 2018 Mar 12. PMID: 29377130.

28. Viaud S, Saccheri F, Mignot G, Yamazaki T, Daillère R, Hannani D, et al, The intestinal microbiota modulates the anticancer immune effects of cyclophosphamide. Science. 2013 Nov 22;342(6161):971-6. doi: 10.1126/science.1240537. PMID: 24264990; PMCID: PMC4048947.

29. https://ecis.jrc.ec.europa.eu/explorer.php? $\$ 0-0 \$ 1-A l l \$ 2-A l l \$ 4-1,2 \$ 3-49 \$ 6-0,85 \$ 5-2008,2008 \$ 7-$ 7\$CEstByCountry $\$ X 0 \_8-3 \$ X 0 \_19-A E 27 \$ X 0 \_20-N o \$ C E s t B y S e x B y C o u n t r y \$ X 1 \_8-3 \$ X 1 \_19-A E 27 \$ X 1$-1- 


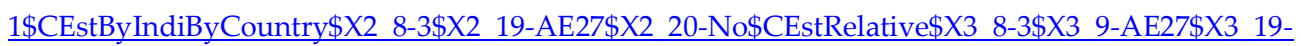
AE27\$CEstByCountryTable\$X4 19-AE27/

30. https://ecis.jrc.ec.europa.eu/explorer.php? $\$ 0-0 \$ 1-A 11 \$ 2-A l l \$ 4-1,2 \$ 3-50 \$ 6-0,85 \$ 5-2008,2008 \$ 7-$ $7 \$$ CEstByCountry $\$ X 0$ 8-3\$X0 19-AE27\$X0 20-No $\$ C E s t B y S e x B y C o u n t r y \$ X 1$ 8-3\$X1_19-AE27\$X1 -11\$CEstByIndiByCountry\$X2_8-3\$X2_19-AE27\$X2_20-No\$CEstRelative\$X3 8-3\$X3_9-AE27\$X3_19AE27\$CEstByCountryTable\$X4_19-AE27

31. https://ecis.jrc.ec.europa.eu/explorer.php?\$0-0\$1-All\$2-All\$4-1,2\$3-51\$6-0,85\$5-2008,2008\$77\$CEstByCountry \$X0 8-3\$X0 19-AE27\$X0 20-No\$CEstBySexByCountry\$X1 8-3\$X1_19-AE27\$X1 -11 1\$CEstByIndiByCountry $\$ X 2 \_8-3 \$ X 2 \_19-A E 27 \$ X 2 \_20-N o \$ C E s t R e l a t i v e \$ X 3$ 8-3\$X3_9-AE27\$X3_19AE27\$CEstByCountryTable\$X4_19-AE27

32. https://ecis.jrc.ec.europa.eu/explorer.php?\$0-0\$1-All\$2-All $\$ 4-1,2 \$ 3-55 \$ 6-0,85 \$ 5-2008,2008 \$ 7-$ 7\$CEstByCountry $\$ X 0 \_$8-3\$X0_19-AE27\$X0 20-No $\$ C E s t B y S e x B y C o u n t r y \$ X 1 \_8-3 \$ X 1$ 19-AE27\$X1_-11 \$CEstByIndiByCountry\$X2 8-3\$X2 19-AE27\$X2 20-No\$CEstRelative\$X3 8-3\$X3 9-AE27\$X3 19AE27\$CEstByCountryTable\$X4 19-AE27

33. https://www.ecdc.europa.eu/en/antimicrobial-consumption/database/quality-indicators

34. Helander HF, Fändriks L. Surface area of the digestive tract - revisited. Scand J Gastroenterol. 2014 Jun;49(6):681-9. doi: 10.3109/00365521.2014.898326. Epub 2014 Apr 2. PMID: 24694282

35. Sochocka M, Donskow-Łysoniewska K, Diniz BS, Kurpas D, Brzozowska E, Leszek J. The Gut Microbiome Alterations and Inflammation-Driven Pathogenesis of Alzheimer's Disease-a Critical Review. Mol Neurobiol. 2019 Mar;56(3):1841-1851. doi: 10.1007/s12035-018-1188-4. Epub 2018 Jun 23. PMID: 29936690; PMCID: PMC6394610.

36. Patterson E, Ryan PM, Cryan JF, Dinan TG, Ross RP, Fitzgerald GF, Stanton C. Gut microbiota, obesity and diabetes. Postgrad Med J. 2016 May;92(1087):286-300. doi: 10.1136/postgradmedj-2015-133285. Epub 2016 Feb 24. PMID: 26912499.

37. Nagpal R, Kumar M, Yadav AK, Hemalatha R, Yadav H, Marotta F, Yamashiro Y. Gut microbiota in health and disease: an overview focused on metabolic inflammation. Benef Microbes. 2016;7(2):181-94. doi: 10.3920/bm2015.0062. Epub 2015 Dec 8. PMID: 26645350.

38. Frazier TH, DiBaise JK, McClain CJ. Gut microbiota, intestinal permeability, obesity-induced inflammation, and liver injury. JPEN J Parenter Enteral Nutr. 2011 Sep;35(5 Suppl):14S-20S. doi: 10.1177/0148607111413772. Epub 2011 Aug 1. PMID: 21807932

39. Nishida A, Inoue R, Inatomi O, Bamba S, Naito Y, Andoh A. Gut microbiota in the pathogenesis of inflammatory bowel disease. Clin J Gastroenterol. 2018 Feb;11(1):1-10. doi: 10.1007/s12328-017-0813-5. Epub 2017 Dec 29. PMID: 29285689.

40. Matsuoka K, Kanai T. The gut microbiota and inflammatory bowel disease. Semin Immunopathol. 2015 Jan;37(1):47-55. doi: 10.1007/s00281-014-0454-4. Epub 2014 Nov 25. PMID: 25420450; PMCID: PMC4281375.

41. Seksik P. Microbiote intestinal et MICI [Gut microbiota and IBD]. Gastroenterol Clin Biol. 2010 Sep;34 Suppl 1:S44-51. French. doi: 10.1016/S0399-8320(10)70020-8. PMID: 20889004

42. Mangiola F, Ianiro G, Franceschi F, Fagiuoli S, Gasbarrini G, Gasbarrini A. Gut microbiota in autism and mood disorders. World J Gastroenterol. 2016 Jan 7;22(1):361-8. doi: 10.3748/wjg.v22.i1.361. PMID: 26755882; PMCID: PMC4698498.

43. Li Q, Zhou JM. The microbiota-gut-brain axis and its potential therapeutic role in autism spectrum disorder. Neuroscience. 2016 Jun 2;324:131-9. doi: 10.1016/j.neuroscience.2016.03.013. Epub 2016 Mar 8. PMID: 26964681

44. Rosenfeld CS. Microbiome Disturbances and Autism Spectrum Disorders. Drug Metab Dispos. 2015 Oct;43(10):1557-71. doi: 10.1124/dmd.115.063826. Epub 2015 Apr 7. PMID: 25852213

45. Caputi V, Giron MC. Microbiome-Gut-Brain Axis and Toll-Like Receptors in Parkinson's Disease. Int J Mol Sci. 2018 Jun 6;19(6):1689. doi: 10.3390/ijms19061689. PMID: 29882798; PMCID: PMC6032048.

46. Sun MF, Shen YQ. Dysbiosis of gut microbiota and microbial metabolites in Parkinson's Disease. Ageing Res Rev. 2018 Aug;45:53-61. doi: 10.1016/j.arr.2018.04.004. Epub 2018 Apr 26. PMID: 29

47. Sampson TR, Debelius JW, Thron T, Janssen S, Shastri GG, Ilhan ZE, et al, Gut Microbiota Regulate Motor Deficits and Neuroinflammation in a Model of Parkinson's Disease. Cell. 2016 Dec 1;167(6):1469-1480.e12. doi: 10.1016/j.cell.2016.11.018. PMID: 27912057; PMCID: PMC5718049.

48. Mulak A, Bonaz B. Brain-gut-microbiota axis in Parkinson's disease. World J Gastroenterol. 2015 Oct 7;21(37):10609-20. doi: 10.3748/wjg.v21.i37.10609. PMID: 26457021; PMCID: PMC4588083. 
49. Vogt NM, Kerby RL, Dill-McFarland KA, Harding SJ, Merluzzi AP, Johnson SC, et al. Gut microbiome alterations in Alzheimer's disease. Sci Rep. 2017 Oct 19;7(1):13537. doi: 10.1038/s41598-017-13601-y. PMID: 29051531; PMCID: PMC5648830.

50. Hu X, Wang T, Jin F. Alzheimer's disease and gut microbiota. Sci China Life Sci. 2016 Oct;59(10):1006-1023. doi: 10.1007/s11427-016-5083-9. Epub 2016 Aug 26. PMID: 27566465.

51. Gerdes LA, Yoon H, Peters A. Mikrobiota und Multiple Sklerose [Microbiota and multiple sclerosis]. Nervenarzt. 2020 Dec;91(12):1096-1107. German. doi: 10.1007/s00115-020-01012-w. Epub 2020 Oct 12. PMID: 33044577

52. Chu F, Shi M, Lang Y, Shen D, Jin T, Zhu J, Cui L. Gut Microbiota in Multiple Sclerosis and Experimental Autoimmune Encephalomyelitis: Current Applications and Future Perspectives. Mediators Inflamm. 2018 Apr 2;2018:8168717. doi: 10.1155/2018/8168717. PMID: 29805314; PMCID: PMC5902007

53. Kovatcheva-Datchary P., Tremaroli V., Bäckhed F. (2013) The Gut Microbiota. In: Rosenberg E., DeLong E.F., Lory S., Stackebrandt E., Thompson F. (eds) The Prokaryotes. Springer, Berlin, Heidelberg. https://doi.org/10.1007/978-3-642-30144-5_87

54. Rajagopala SV, Vashee S, Oldfield LM, Suzuki Y, Venter JC, Telenti A, Nelson KE. The Human Microbiome and Cancer. Cancer Prev Res (Phila). 2017 Apr;10(4):226-234. doi: 10.1158/1940-6207.CAPR-16-0249. Epub 2017 Jan 17. PMID: 28096237.

55. Picardo SL, Coburn B, Hansen AR. The microbiome and cancer for clinicians. Crit Rev Oncol Hematol. 2019 Sep;141:1-12. doi: 10.1016/j.critrevonc.2019.06.004. Epub 2019 Jun 8. PMID: 31202124

56. Song Y, Gyarmati P. Microbiota changes in a pediatric acute lymphocytic leukemia mouse model. Microbiologyopen. 2020 Mar;9(3):e982. doi: 10.1002/mbo3.982. Epub 2019 Dec 29. PMID: 31884727; PMCID: PMC7066458.

57. Uribe-Herranz M, Klein-González N, Rodríguez-Lobato LG, Juan M, de Larrea CF. Gut Microbiota Influence in Hematological Malignancies: From Genesis to Cure. Int J Mol Sci. 2021 Jan 20;22(3):1026. doi: 10.3390/ijms22031026. PMID: 33498529; PMCID: PMC7864170.

58. DiDonato JA, Mercurio F, Karin M. NF- $\mathrm{BB}$ and the link between inflammation and cancer. Immunol Rev. 2012 Mar;246(1):379-400. doi: 10.1111/j.1600-065X.2012.01099.x. PMID: 22435567.

59. Musolino C, Allegra A, Innao V, Allegra AG, Pioggia G, Gangemi S. Inflammatory and Anti-Inflammatory Equilibrium, Proliferative and Antiproliferative Balance: The Role of Cytokines in Multiple Myeloma. Mediators Inflamm. 2017;2017:1852517. doi: 10.1155/2017/1852517. Epub 2017 Sep 26. PMID: 29089667; PMCID: PMC5635476

60. Yu H, Lee H, Herrmann A, Buettner R, Jove R. Revisiting STAT3 signalling in cancer: new and unexpected biological functions. Nat Rev Cancer. 2014 Nov;14(11):736-46. doi: 10.1038/nrc3818. PMID: 25342631.

61. Li D. Diabetes and pancreatic cancer. Mol Carcinog. 2012 Jan;51(1):64-74. doi: 10.1002/mc.20771. PMID: 22162232; PMCID: PMC3238796.

62. Rajagopala SV, Yooseph S, Harkins DM, Moncera KJ, Zabokrtsky KB, Torralba MG, Tovchigrechko A, Highlander SK, Pieper R, Sender L, Nelson KE. Gastrointestinal microbial populations can distinguish pediatric and adolescent Acute Lymphoblastic Leukemia (ALL) at the time of disease diagnosis. BMC Genomics. 2016 Aug 15;17(1):635. doi: 10.1186/s12864-016-2965-y. PMID: 27527070; PMCID: PMC4986186.

63. Cozen W, Yu G, Gail MH, Ridaura VK, Nathwani BN, Hwang AE, Hamilton AS, Mack TM, Gordon JI, Goedert JJ. Fecal microbiota diversity in survivors of adolescent/young adult Hodgkin lymphoma: a study of twins. Br J Cancer. 2013 Mar 19;108(5):1163-7. doi: 10.1038/bjc.2013.60. Epub 2013 Feb 26. PMID: 23443674; PMCID: PMC3619077.

64. Song Y, Himmel B, Öhrmalm L, Gyarmati P. The Microbiota in Hematologic Malignancies. Curr Treat Options Oncol. 2020 Jan 11;21(1):2. doi: 10.1007/s11864-019-0693-7. PMID: 31927673.

65. Ramirez J, Guarner F, Bustos Fernandez L, Maruy A, Sdepanian VL, Cohen H. Antibiotics as Major Disruptors of Gut Microbiota. Front Cell Infect Microbiol. 2020 Nov 24;10:572912. doi: 10.3389/fcimb.2020.572912. PMID: 33330122; PMCID: PMC7732679. 
